\title{
Frontières
}

\section{Entre dislocation et lien}

\section{Entretien avec Michèle Lepeer}

\section{Alice Verstraeten}

Volume 19, numéro 1, automne 2006

Enjeux politiques et mort

URI : https://id.erudit.org/iderudit/016646ar

DOI : https://doi.org/10.7202/016646ar

Aller au sommaire du numéro

Éditeur(s)

Université du Québec à Montréal

ISSN

1180-3479 (imprimé)

1916-0976 (numérique)

Découvrir la revue

Citer ce document

Verstraeten, A. (2006). Entre dislocation et lien : entretien avec Michèle Lepeer. Frontières, 19(1), 105-108. https://doi.org/10.7202/016646ar

Ce document est protégé par la loi sur le droit d'auteur. L'utilisation des services d'Érudit (y compris la reproduction) est assujettie à sa politique d'utilisation que vous pouvez consulter en ligne.

https://apropos.erudit.org/fr/usagers/politique-dutilisation/
Cet article est diffusé et préservé par Érudit.

Érudit est un consortium interuniversitaire sans but lucratif composé de l’Université de Montréal, l'Université Laval et l'Université du Québec à Montréal. Il a pour mission la promotion et la valorisation de la recherche. https://www.erudit.org/fr/ 


\section{Entre dislocation et lien Entretien avec Michèle Lepeer}

\section{Propos recueillis par Alice Verstraeten, anthropologue et fille de l'artiste.}

Il ne suffit pas de connaître les œuvres d'un artiste, il faut aussi savoir quand il les faisait, pourquoi, comment, dans quelles circonstances. Sans doute existera-t-il un jour une science que l'on appellera peut-être la «science de l'homme », laquelle cherchera à pénétrer plus avant l'homme à travers l'homme créateur'.

\section{Quel serait le but de ta peinture?}

De dénoncer! Je suis une petite fille qui n'a jamais pu supporter l'injustice, moi! C'est quand même ça le gros moteur, je crois. Je n'ai jamais compris pourquoi tout le monde n'avait pas la même chance. Pourquoi on parlait des «Noirs» en termes de "sous-hommes", pourquoi on parlait des Flamands comme des "paysans » [Rire] et des francophones comme des «méprisants».

Je me sens anéantie quand je vois les informations, des sorties de camps par exemple. Ça me donne envie de vomir. Je me demande comment faire, comment faire pour résister, comment faire pour exorciser. Je suis assez obsédée par la... répétition des choses, la répétition de l'histoire. Pourquoi est-ce que les humains ont besoin de faire souffrir l'autre, de le torturer?

\section{Ce que tu dis est tellement viscéral que, tu le sais, beaucoup de gens nous demandent si tu es " survivante » et de quoi...}

Survivante. [Silence] Je devais avoir douze ans quand j'ai vu la sortie des camps à la télé. On n'avait pas de télé à la maison, j'étais chez Philippe, un cousin. Je ne sais pas ce que j'ai ressenti, je n'en sais rien, j'étais... Ça paraissait impossible! Je les ai souvent entendu dire "L'homme est un loup pour l'homme", dans la famille. [...] Je ne savais pas du tout qu'on avait des origines juives, mais j'ai dû sentir Philippe très... touché. C'est lui qui avait dit à la famille de ne pas aller chercher l'étoile jaune. Il avait compris, lui, que l'étape suivante c'était les camps.

Je devais avoir vingt ans quand une autre cousine m'a parlé. Elle était survivante des camps, elle était mal physiquement, elle avait des séquelles. Je ne sais plus trop quoi. C'est le flou complet, je ne me souviens pas du tout de ce qu'elle a raconté. Je me souviens juste de l'endroit où elle était assise et de la lumière qu'il y avait. [...]

Je crois que je peins par nécessité humaine. Que ça remplit mon vide, l'angoisse de la solitude. J'aime beaucoup ces vers de Mahmoud Darwich:
"Seuls, nous sommes seuls jusqu'à la lie S'il n'y avait la visite des arcs-en-ciel ${ }^{2}$ »

Ce peut même être la solitude de tout un peuple. On peut être nombreux et très seuls. J'aime l'idée que la peinture est un arc-enciel, même si ça a l'air un peu bête.

\section{Et pourquoi est-ce que c'est la peinture que tu as choisie?}

[Silence] Les mots je m'en méfie, moi. [Silence]

\section{Pourquoi?}

Pourquoi ?! [Rire] Pourquoi je me méfie des mots? À cause des mensonges, des nondits. Un mot peut vouloir dire une chose et son contraire. Selon les gens qui le prononcent, ce n'est pas la même chose non plus. Les mots sont liés à la politique, pour moi. Marx, sur le papier, c'était intéressant. Comme beaucoup de jeunes de ma génération, je me suis fait avoir par le maoïsme pendant un temps, j'ai cru à cette illusion du «petit livre rouge». Une illusion qui devait permettre à quelques-uns de devenir tout-puissants. Les idéologies et le politique se collapsent trop vite pour développer le pouvoir de quelques-uns. Homicide, génocide, on est reparti. C'est aussi pour ça que les mots me font peur. Avec des mots, on harangue des foules, on crée des masses. Et 
si l'homme n'existe plus en tant que sujet, il n'a plus d'humanité.

\section{Mais pour l'image, photographique au moins, le sens peut aussi être manipulé...}

Oui. Le matraquage des images, par exemple, le vide de leur sens humain, de leur sens de vie. Ce qui me frappe dans les photos, c'est leur côté glacé, "figé». Tout y est... sur le même plan. Je ne suis pas sûre que les gens aillent "plus au fond», ils regardent la photo de façon esthétique.

J'essaie d'être «juste» en portant mon attention sur de petits détails. Par exemple, la photo du Honduras, de laquelle je suis partie pour ma toile, m'avait frappée à cause de la main de ce gamin, avec sa petite bague, son petit geste délicat de petit enfant. Il a un pantalon pareil à ceux des petits garçons d'ici. Si on découpait le petit gamin, je ne pense pas qu'on imaginerait qu'il soit occupé à fouiller une décharge puante. Il fallait trouver une lumière qui fasse qu'on le perçoive vraiment comme un enfant qui pourrait être le sien, celui du voisin. Que ce soit ça qui marque. Sur les images des revues, quelque part, c'est exotique. Les gens ne se sentent pas concernés. [...]

Je regarde les photos très longtemps, pour me jeter dedans. À partir de la photo, je recherche... les détails qui croisent des réalités, qui font que ça pourrait être chez nous et que ça pourrait toucher les gens. C'est la même chose avec le cadenas des prisonniers talibans. [Elle ferme les yeux pour décrire sa toile.] Tous les prisonniers sont alignés, comme dans un poulailler. Et le cadenas qui tient la chaîne qui les enferme, c'est un cadenas vert, le plus universel qui soit, je pense. Ici, les gamins utilisent ces cadenas pour fermer leurs casiers à l'école.

\section{Tu crois que ta peinture peut avoir un rôle politique ou un rôle face au politique?}

Je ne sais pas. J'aimerais bien, évidemment. J'aimerais servir de grain de sable pour que l'on puisse voir les choses autrement. J'en reviens toujours à cet aphorisme de Mizogushi: «Il faut se laver les yeux entre chaque regard.» $\mathrm{Si}$ on pouvait se laver les yeux entre chaque journal télévisé... Parce qu'on nous met des œillères, des lunettes qui normalisent le regard. C'est toujours le même cadre, la même lumière. Ce sont les images qui sont lavées, pas les regards. On gomme les détails. Même les images de pub sont «nettoyées », déshumanisées... Même si c'est anodin et que ce n'est pas dans la violence.

C'est très compliqué d'être juste. C'est bien pour ça que je redoutais d'être confrontée à des victimes. Je ne veux pas que mes toiles soient «récupérées » (par Poutine, tu imagines?!). J'ai un peu peur d'en rajouter dans la souffrance, j'ai peur du voyeurisme. Il y a cette crainte que ça se retourne contre les idées que je veux transmettre.

Lors de ma première confrontation avec des victimes [colombiennes, rwandaises, entre autres], j'ai eu l'impression que ça leur faisait «plaisir», parce qu'elles savaient, comme ça, qu'on pense à elles, que ce ne sont pas des oubliées. Primo Levi le disait, que «le sentiment de notre existence dépend pour une bonne part du regard que les autres portent sur nous ${ }^{3} »$.

Je crois, d'autre part, qu'il n'y a rien de pire que l'oubli. J'ai lu un jour cette phrase de Jélu Jélev, qui disait qu'avant de tourner une page, il faut la lire ${ }^{4}$. Il faut parler du passé, le formuler, l'exprimer. Mais pas pour oublier ensuite. Savoir que d'autres y ont prêté attention, ça peut être une petite goutte d'eau pour aider à ce que les gens ne soient pas seuls.

J'aime aussi la peinture parce que j'aime les savoir-faire, et puis parce que c'est quelque chose que tout le monde peut comprendre un peu. Et essayer de faire quelque chose qui techniquement ne soit pas bâclé, qui soit «bien fait », cela me paraît un minimum de respect que je dois aux gens que je peins. [...]

[Long silence] J'ai l'impression de m'inscrire un petit peu dans la longue tradition des peintres fresquistes. J'aurais aimé faire des fresques, moi. Je n'ai pas dit mon dernier mot, d'ailleurs! J'aimerais que la peinture rentre plus dans la sphère publique qu'elle ne l'est. Parce qu'avant, comme avec Michel-Ange, elle était dans les Églises... Je ne suis pas en train de dire que je me prends pour Michel-Ange! Mais la peinture peut transmettre un message collectif. [...] Je dis toujours qu'il y a différentes manières d'être peintre. Il y a des peintres décoratifs, des peintres de l'armée, des peintres animaliers... "Peintre», ça ne veut rien dire. Ça ne suffit pas en tous cas. [Elle s'éclaircit la voix] Moi, j'aimerais être peintre militant... c'est un peu «lourd»...euh... Oui, je trouve que ça peut compléter une parole, une logique, un discours. Ignacio Ramonet ${ }^{5}$ disait qu'il y a différents types de peintres: les Narcisse et les Prométhée. Je me sens évidemment plutôt Prométhée. J'ai toujours été fascinée par ce personnage-là. [...] Et si je fais une peinture relativement réaliste, je pense qu'il y a beaucoup de gens qui peuvent passer, rentrer dedans. On entend toujours les gens dire «l'art moderne, l'art contemporain, j'y comprends rien ». À mon sens, ce n'est pas la fonction de l'art que d'entendre tout le monde dire ça.

\section{Tu as toujours peint I'apparition/ disparition. Même sans peindre ces sujets-là.}

Oui. Des choses comme ça. Et ça revient même assez fort. J'essaie de travailler autrement techniquement, plus «sur le lit de la couleur». La toile va devenir plus "unie», et je cherche à ce que les choses se dégagent de la couleur de la toile.

Quand je peignais des sujets d'eau, je me souviens avoir dit une fois que l'eau me permettait de diffracter, de composer les choses, que ce ne soit pas figé, qu'il y ait du mouvement... Et puis l'eau, c'est le temps qui passe. C'est un peu lourd en symbolique, mais... J'ai travaillé le temps qui passe aussi, beaucoup. J'ai travaillé le mouvement, les choses qui apparaissent et qui disparaissent. Je crois que la mort est là, dans mon histoire d'apparitions/disparitions. Peindre des oiseaux, par exemple, peindre les mouvements de l'eau, c'est représenter la mémoire d'un passage dans l'œil plutôt que le passage lui-même. C'est une palpitation de vie. Le temps, c'est la vie, mettre les choses en mouvement, c'est représenter le vivant. Avec la peinture, je prends le temps. C'est un «passe-temps »! [Rire]

C'est aussi pour cela que les mots ne me satisfont pas: parce qu'avec eux les choses disparaissent trop vite. Tu crois toucher à quelque chose de juste et hop, ça file. La peinture, elle, est autonome. Viscérale. En peignant, je dis ce que je sais, mais aussi ce que je ne sais pas.

[...] Après l'eau, j'ai fait des charniers. C'était peindre la fragilité du corps, entre dislocation et lien. C'est compliqué. [...] Après je suis passée aux «déchirés » : déchirer des photos pour faire des collages. C'était une autre manière de disloquer. Je représentais sans que ce soit une image plate. J'avais fait toute une série de peintures avec des papiers chiffonnés aussi. Des drapés, des chiffonnés, c'est un peu la même idée. [...]

\section{Et ce passage au charnier, comment s'est-il dessiné?}

J'ai toujours peint des corps humains. Toujours, toujours, toujours. Pour moi, il faut un être humain sur une peinture (un corps humain, souvent nu, comme dans la Sixtine). [...] J'avais repris un Goya il y a très, très longtemps. Au pastel. Si je reprends des gravures que j'ai faites quand j'étais étudiante, on en retrouve comme ça... Des corps qui tombent. J'ai beaucoup fait des choses comme ça. J'ai peint des trapézistes, pour mettre les corps dans l'espace. Il y a eu quelques Icare, aussi. C'était, en plus, une petite référence à Bruegel. Cela m'a toujours impressionnée qu'il puisse montrer 


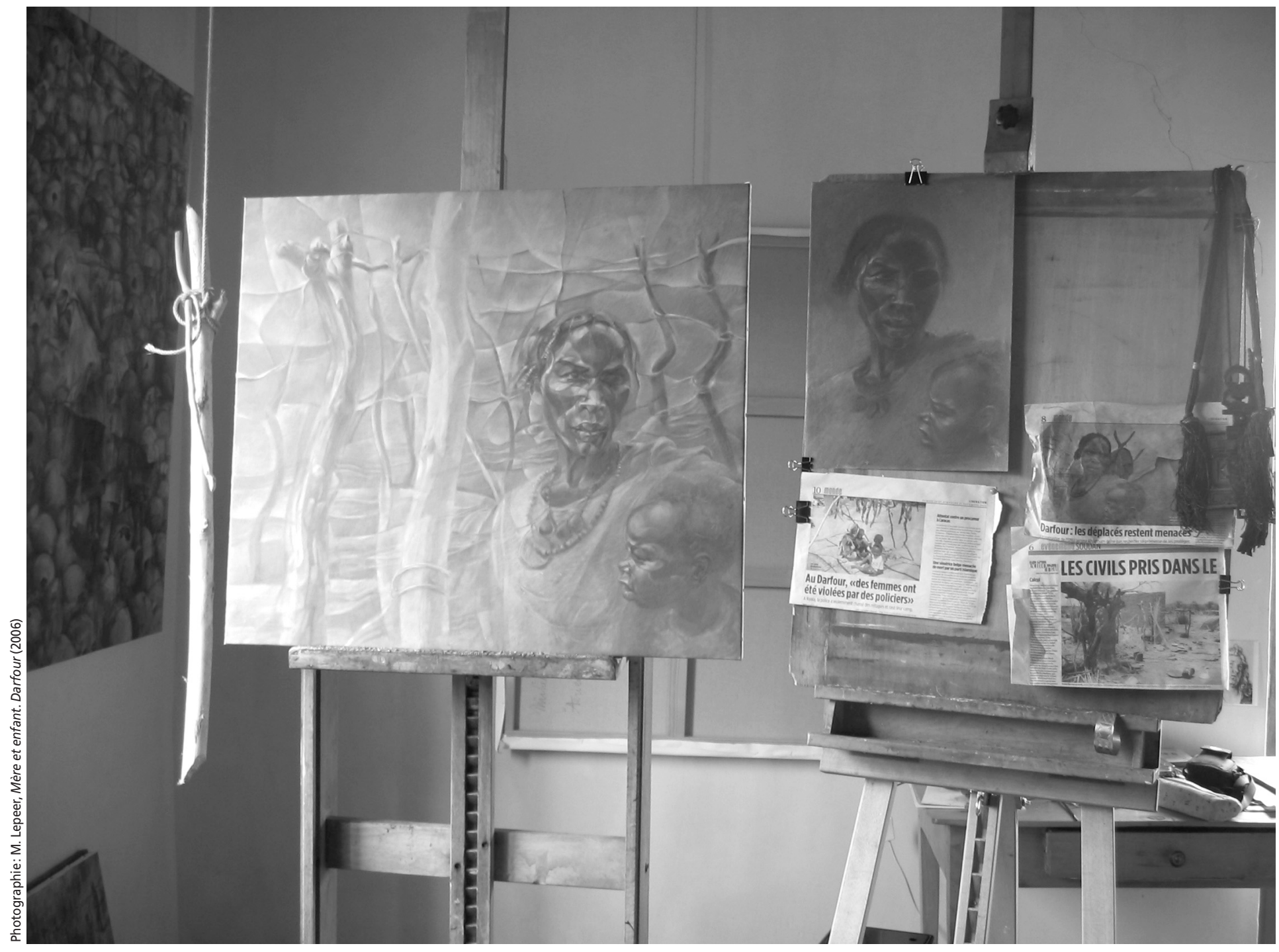

Photographie du studio de Michèle Lepeer. On y voit l'œuvre Mère et enfant, Darfour (2006) en production, entourée d'éléments aidant à la réalisation.

Icare, alors qu'il n'est qu'une anecdote dans son tableau. C'était une belle manière de raconter une histoire.

Mais je ne savais pas comment les aborder, ces sujets-là. [...]

\section{Dans mes souvenirs, c'est quand tu faisais tes dialogues avec Michel- Ange qu'ont commencé à apparaître dans tes tableaux des femmes hurlant ou des...}

Mmmh. Parce qu'en fait je me suis rendue compte, en regardant la Sixtine, que si je la sortais complètement de son contexte biblique, c'était presque de l'illustration de ce qui se passe dans le monde en général... [Silence] Il y a une douleur là-dedans qui est... intemporelle. C'est un exercice que je conseille à tout le monde, de regarder la Sixtine en la sortant de son contexte. C'est assez étonnant. Parce que si on prend des petits morceaux des expressions, si on voit l'angoisse qui se lit dans les regards, c'est... c'est fascinant. Je n'ai pas eu de mal du tout à introduire de l'actualité là-dedans.

\section{Quand tu t'es "libérée " de Michel-Ange, c'était à partir d'un événement précis ?}

C'était la Bosnie. C'était à partir de la Bosnie. Cela devait faire répétition de beaucoup de choses, là, dans la conscience européenne. [Petit rire] Ces préoccupations étaient présentes quelque part dans ma tête, mais je crois que c'est surtout la Bosnie qui a... réveillé la guerre de 40 : on a revu des photos qui rappelaient vraiment les camps de concentration, les disparus, les non-dits. Alors que l'on avait dit «plus jamais ça» et que, quelque part, on dormait sur nos deux oreilles. Dans notre génération, on avait eu l'impression que ça ne reviendrait pas, que le fait d'en parler faisait de cette barbarie une barbarie d'un autre temps. [Silence]

Et ce qui a beaucoup, beaucoup, beaucoup joué... [Silence] c'est le Rwanda, c'est une émission radio de Daniel Mermet qui découvrait la petite Valentine, survivante mutilée d'un massacre. Le Rwanda aussi est un rappel de l'histoire. Les responsabilités belges sont... énormes.

Après, dans mes peintures, ça s'est enchaîné... ou déchaîné, si on préfère!

\section{Tu pourrais raconter l'élaboration de tes toiles?}

Il y a plusieurs sujets qui tournent dans ma tête. J'aimerais bien les travailler mais je ne sais pas encore comment. Et alors ça se construit petit à petit avec des rencontres d'images, de choses que je vois, que j'entends ou que je peux lire. Quand j'ai plusieurs documents, j'étale tout, et des choses 
en sortent. Une fois que je pense que c'est bon, que je tiens le sujet à peu près, alors je démarre avec un ou deux croquis. Je fait des portraits pour essayer de vraiment... trouver le regard. Ça, c'est important. [...] Après les croquis, parfois, je fais un pastel, puis je peux faire soit gravure, soit un lavis à la plume, une aquarelle, une acrylique. J'aime bien varier les techniques, parce que cela m'évite d'être trop systématique. Parce qu'une technique peut en pousser une autre. Les différents sujets n'appellent pas tous la même technique: si c'est très, très "graphique », je vais être attirée au départ par un travail à la plume, si c'est une question de couleurs je vais plutôt partir sur du pastel. Et une fois que tout est prêt, quand je pense avoir résolu toutes les difficultés techniques éventuelles, je me jette dans ma toile. Je suis, par exemple, en train de préparer une burka. J'ai fais un dessin préparatoire très minutieux du voile de la burka. J'ai essayé de comprendre comment se tissait le coton du petit emplacement qui permet aux femmes de voir. Parce que je veux être très juste là-dedans.

\section{Depuis que tu peins ce genre de sujets, tu as l'impression d'avoir évolué?}

[Silence] Oui, ça a pas mal changé. [Silence]. Je pense que je me sentais agressée par ces images, et que quelque part je rendais cette agression. Quelque part. Or, je ne suis pas sûre que ce soit nécessairement la bonne piste. Je voudrais bien arriver à ce que les gens puissent regarder. Et pensent l'autre autrement que celui qui est soit exotique, soit... soit " pas du même niveau d'humanité».

J'ai l'impression que quelque chose de "plus humain» vient dans la sensualité. Évidemment, en faisant des peintures de charniers, il est difficile d'être « sensuel »... Mais, par exemple, dans cette peinture que j'ai faite sur Srebrenica, il y a ce tissu, ce tricot qui est sur le haut des chevilles, qui pour moi représentait... le fait que sa mère, sa femme ou sa sœur lui avait tricoté une écharpe. Au dernier moment, il a essayé de se tenir chaud, encore un peu, avec ça. Et il s'est enveloppé les pieds. Et c'est la seule chose qui donne une histoire humaine à ce squelette 6 .

\section{Mais pourquoi dis-tu que tu « rendais de l'agressivité"», au début?}

[Silence] De l'agressivité... Si ce n'est pas regardable du tout, ce n'est pas intéressant. Le but est quand même... de faire réfléchir, ou de faire aller vers l'autre comme alter ego et non comme un autre "différent». [...] Or, un charnier à l'état brut est un "coup de poing », un choc. C'est bien d'en faire comme ça, aussi. Mais je ne suis pas sûre que ça suffise pour que le regard soit dans l'échange, justement. Selon moi, la peinture sert d'intermédiaire vers la personne qui regarde. Si c'est répulsif, c'est raté.

Ces derniers temps, j'en reviens beaucoup à l'idée de la «caresse». Peut-être que l'idée est venue avec les «Mères de la place de Mai ». Parce que vraiment j'ai une admiration sans limites pour elles. Parce qu'elles ont ... elles, elles ont résisté avec de la tendresse, avec de l'affectif, elles font mémoire. C'est aussi le rôle de la peinture, ça. La peinture, c'est un objet qui reste, qui peut se revoir, qui n'est pas comme une feuille de papier. Je ressens comme une tendresse infinie, quelque chose comme ça.

Il reste des gens qui ne mettent pas les pieds dans l'atelier. [Silence] Mais de plus en plus, les gens regardent mes toiles, ils prennent le temps de le faire, et s'ils sont deux ou trois ils commencent à discuter. Ce qui me frappe, c'est qu'ils disent beaucoup de choses qui n'ont rien à voir avec le sujet de départ. C'est vrai que mes toiles sont assez complexes, qu'il y a beaucoup de choses à regarder. J'aime bien qu'il y ait dans mes toiles des détails dans lesquels les personnes peintes puissent se reconnaître, et en même temps que les autres puissent y voir autre chose et se «promener» dedans. Il y a par exemple eu la maman d'un petit voisin, qui ne connaît pas du tout la peinture, qui n'a jamais vu d'exposition. Comme tout le monde, elle a une affiche d'un impressionniste chez elle, mais c'est tout. En venant chercher son fils, elle a fait un petit tour dans l'atelier. Je l'ai revue deux jours après et elle m'a dit: «Tu sais que toute la journée, après, j'y ai repensé ? » Non seulement elle aimait bien la technique, mais ça l'avait bouleversée. Et elle n'avait pas l'air de le regretter du tout. Je ne l'ai pas rendue malade, elle était contente d'avoir rencontré ça. Dans cette évolution de ma peinture, je suis peut-être arrivée tout doucement à quelque chose qui ressemble à mes intentions.

Dimanche soir, après une exposition avec Amnistie, Jean - un ami en fin de vie, qui essaie de partir dignement - a touché ma toile sur Guantanamo, pour lui dire au revoir. C'est fantastique, cette envie de la toucher. D'un côté, tu deviens toi-même avec ton travail et de l'autre côté, tu vois le chemin que ta peinture peut faire toute seule.

\section{Notes}

1. Confidences de Picasso à Brassaï (déc. 1943), dans «Les 13 journées de vie de Picasso», documentaire de P.-A. Bontang, P. Daix et P. Philippe, 1999.

2. M. Darwich (avril 2002). "Poème Inédit», dans Le Monde diplomatique.

3. P. Levi (1987). Si c'est un homme, Paris, Julliard, Pocket, «Appendice», p. 191.

4. Propos repris par T. Todorov (déc. 1999), p. 18, «Mémoire du mal », dans Courrier de l'UNESCO, p. 18-19.

5. I. Ramonet (1999). Géopolitique du chaos, Paris, Gallimard, Folio.

6. Voir les pages 10-11 du présent numéro de Frontières. 